\title{
Blockchain in Agriculture Traceability Systems: A Review
}

\author{
Konstantinos Demestichas*(D), Nikolaos Peppes, Theodoros Alexakis and Evgenia Adamopoulou \\ Institute of Communication and Computer Systems, Zografou, 15773 Athens, Greece; npeppes@cn.ntua.gr (N.P.); \\ talexakis@cn.ntua.gr (T.A.); eadam@cn.ntua.gr (E.A.) \\ * Correspondence: cdemest@cn.ntua.gr; Tel.: +30-210-772-1478
}

Received: 6 May 2020; Accepted: 10 June 2020; Published: 15 June 2020

Featured Application: The paper elaborates on the applicability of blockchain technology in traceability systems of agri-food products.

\begin{abstract}
Food holds a major role in human beings' lives and in human societies in general across the planet. The food and agriculture sector is considered to be a major employer at a worldwide level. The large number and heterogeneity of the stakeholders involved from different sectors, such as farmers, distributers, retailers, consumers, etc., renders the agricultural supply chain management as one of the most complex and challenging tasks. It is the same vast complexity of the agriproducts supply chain that limits the development of global and efficient transparency and traceability solutions. The present paper provides an overview of the application of blockchain technologies for enabling traceability in the agri-food domain. Initially, the paper presents definitions, levels of adoption, tools and advantages of traceability, accompanied with a brief overview of the functionality and advantages of blockchain technology. It then conducts an extensive literature review on the integration of blockchain into traceability systems. It proceeds with discussing relevant existing commercial applications, highlighting the relevant challenges and future prospects of the application of blockchain technologies in the agri-food supply chain.
\end{abstract}

Keywords: blockchain; distributed ledger; traceability; agriculture supply chain; agri-food industry

\section{Introduction}

Food supply chains have a vast complexity which is often the cause of lack of transparency and traceability. On top of that, a major issue directly affecting public health is food safety. During the past twenty years, various food epidemic incidents have been reported, like the foot-and-mouth Disease in Europe in 2001, the Escherichia coli outbreak in spinach in 2006 in USA, the Sanlu milk scandal in China in 2008, the E. coli O104:H4 outbreak in Germany in 2011, the South African listeriosis outbreak in 2017-2018, etc. Governments and health organizations, in an attempt to prevent such dangerous outbreaks, have established relevant directives, laws as well as standards and regulations. For example, in Europe, the traceability of food products is compulsory according to the European Directive 178/2002 since 1 January 2005, together in compliance with the HACCP (Hazard Analysis and Critical Control Points) principles. Likewise, regulations all over the world have been established aiming to diminish food epidemic incidents.

Nowadays, consumers' concerns regarding food provenance and quality are extremely high, resulting in the tendency to spend more money on food products whose origin is certified. Despite the developed technologies that are already in use, in many cases, the vast majority of the traceability systems are centralized, asymmetric and outdated in terms of data sharing and interoperability. Existing systems lack in transparency and consumers' trust due to the unavailability of a fast 
and trustworthy way to retrieve information on the product's provenance. Considering all the above, together with the rapid technological development adopted in value chain areas, we observe a significant increase in emerging innovations that lead the way for new digital traceability systems by taking advantage of information and communication technology (ICT), Radio-Frequency Identification (RFID) sensors, Internet of Things (IoT), blockchains and more. In this context, distributed ledger technologies (DLT) such as blockchain offer a solution to many existing problems, but simultaneously pose new challenges as well.

Over the last few years, a rapid increase regarding the practical and effective application of DLT in the agri-supply chain has been under the spotlight in the academic and food industry communities [1]. In addition, the establishment of the Industry 4.0 paradigm renders DLT a promising area for further research in the future. In this paper, the authors identify, gather and present a thorough literature review of ongoing scientific papers, technical publications, research projects and pilot integrated and commercial platforms using blockchain for traceability in the agriculture supply chain. The main target of this paper is to conduct an analysis of the research activities performed over the last years and demonstrate a taxonomy of different ideas and a chronological presentation of useful studies. This will serve as the basis for conducting further research in order to better address the challenges that still exist in the domain.

The remainder of the paper is organized as follows: Section 2 presents an overview of the definitions, levels, tools and advantages of traceability; Section 3 provides a brief review about the blockchain technology; Section 4 includes a literature review of related activities to date regarding the integration of blockchain into traceability systems; Section 5 presents relevant existing commercial applications; Section 6 highlights challenges and future prospects; Section 7 concludes the paper.

\section{Traceability}

\subsection{Literature Review of Traceability—Definitions and Categorizations}

Traceability, also known as the principle of "one step back one step forward", is the ability to recall all the information about the origin of a food product [2]. Another definition of traceability, given by the International Organization for Standardization (ISO) in ISO 22005:2007, is the "ability to follow the movement of a feed or food through specified stage(s) of production, processing and distribution" [3]. The European Union General Food Law EC 178/2002 defines traceability as the ability, in all production, processing and sales stages, of tracing and tracking the food [4]. An overview of the product and information flows in a typical traceability system is depicted in Figure 1.
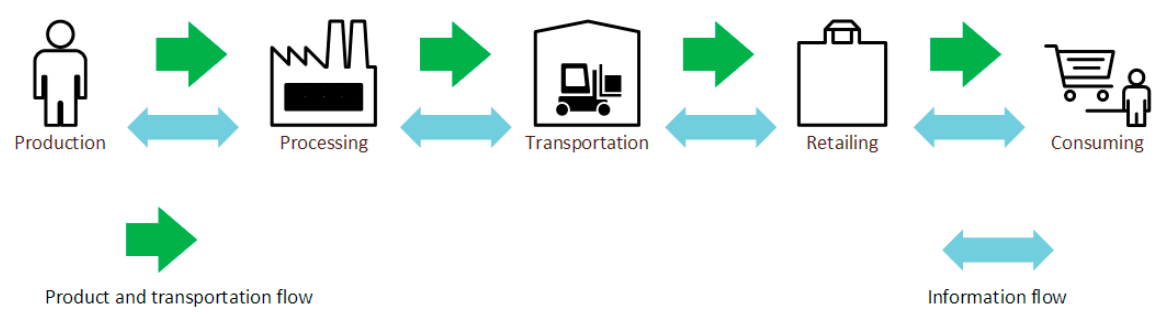

Figure 1. Traceability system product and information flow.

Considering all the above, food product traceability contains information about the food ingredients, the food sources, processing as well as transportation and storage conditions. An ideal agriculture traceability system would also contain information about each and every ingredient of the final product. Thus, to be considered effective, a traceability system must contain information, both quantitative and qualitive, about the final food product and its origin. According to A. Corallo et al., there are four major questions that need to be answered regarding the development of a traceability system [5]:

1. Which data are important and must be collected? 
2. Who is the owner of the information in every stage of the supply chain?

3. Which tools must be used for data collection (sensors, network, etc.)?

4. How should the data be managed in order to be available and understandable for both the stakeholders and consumers?

Corallo et al. and Aung et al. identify two different levels of traceability:

1. The intra-company (internal) level;

2. The supply chain or external level.

Specifically, the internal level traceability is carried out by a single actor (company, organization, etc.) and through internal procedures [5,6]. Furthermore, intra-company traceability tries to identify the origin of the product's ingredients, packaging, etc., in case this actor of the supply chain is asked. On the other hand, the external traceability is a combination of intra-company processes and a reconstructing procedure of the whole history of the specific product [6]. Subsequently, all the stakeholders in a supply chain must be involved and coordinate with each other in order to produce a trustworthy outcome in terms of traceability.

Another categorization regarding the traceability level has been performed by the European Community market, which distinguishes the mandatory traceability and the voluntary traceability $[5,7]$. The mandatory traceability mainly has to do with financial purposes and lacks detailed information about the products regarding quality aspects. Supplementary to mandatory traceability, voluntary traceability refers to the ability of every actor on the supply chain to freely select what data to gather [5]. Justifying its name, voluntary traceability is not compulsory for stakeholders to implement. Trustworthy and complete traceability is only possible when both mandatory and voluntary traceability processes exist. Stakeholders involved in the supply chain enable a more detailed and qualitive traceability system when they voluntarily add information. A main challenge for voluntary systems lies in their complexity, since every actor may have their own standards and methods for tracking and tracing a product, leading to a wide variety of acquired data [8].

\subsection{Agriculture Traceability Tools and Technology Solutions}

Agriculture traceability demands a large volume of data that needs to be collected across the supply chain. Very early tracking and traceability systems used workers to record the information on the field and then manually transfer them on handbooks or into a computer system. This method entails risks, such as faulty information recording as well as inefficient resource usage. In the last recent decades, there is a rapid development of automated processes and products, as well as in communication technologies, resulting in the so-called Internet of Things (IoT) paradigm. This rapid evolution of IoT and sensor technology favors the data gathering procedure by offering fast and reliable methods. These methods include technology for product identification, ingredient analysis, transportation, storage, as well as information capture throughout the overall system integration. Methods such as barcodes, QR codes, RFID, wireless sensor networks (WSNs) are the most widespread and well-known among supply chains.

Due to their attributes, RFID systems provide a safe information and data management system of agri-food for producers, wholesalers, retailers and consumers. RFID technology contributes in agri-food supply chain management by tracing and monitoring of the "from farm to fork" route. Once a food safety issue occurs, its source and consequently the solution can rapidly be found $[9,10]$.

An alternative or complement to RFID tags are wireless sensors networks (WSNs). A WSN consists of wireless sensors and actuators, such as humidity sensors, temperature sensors, sound sensors, pressure sensors, regulators and more, which communicate via a wireless modem with an external system where the data are stored and rendered available for the stakeholders. Through WSNs, information, such as temperature, humidity, wind speed, plant diseases, insect pests and animal vital signs, can be obtained, processed and sent to a higher level for decision support. WSNs usually consist of many sensor nodes which are battery powered and have low energy consumption. 
Specifically, the sensors in agriculture are usually planted into soil, in order to collect data regarding the plantation conditions [11]. Moreover, WSNs can be combined with more advanced technologies such as GPS and remote sensing to provide extra functionality.

As the food supply chain is extremely complex and, at the same time, consumer needs and awareness for fresh, safe and quality food are continuously growing, traceability systems offer many advantages, including the decrease in the time needed to recall and withdraw products dangerous for the public health, thus improving consumers' safety and confidence. Furthermore, traceability provides the agriculture supply chain with transparency and reliability, attributes of outmost importance considering the complexity of the food supply chain nowadays.

\section{Blockchain}

Hence, traceability systems address a range of issues, including food fraud, food security and withdrawal, compliance with regulations, societal issues and consumer awareness [12]. For businesses, tracing and tracking products can lead to increased value of business assets and to increased profit through costs reduction in the long term.

\subsection{Overview and Definitions}

Some groundwork for what we nowadays refer to as "blockchain" was performed by Stuart Haber and W. Scott Stornetta in 1991, in their article entitled "How to Time-Stamp a Digital Document" [13], while the popular paper by Satoshi Nakamoto in 2008 [14] established bitcoin as a cryptocurrency and devised the first blockchain database. According to Mayank Raikwar et al., a very general definition of blockchain is: "Blockchain is a distributed ledger maintaining a continuously growing list of data records that are confirmed by all of the participating nodes" [15].

A block is a record that includes data inside it, as well as a value with the previous block's hash and, finally, a value that represents its own hash. The hash stands for the digital fingerprint of an amount of data of the block. The link between the hash of the current block and the hash of the previous block explains the meaning of the cryptographically linked chain of blocks through these hashes. If anyone tampers with the data, this digital fingerprint will be changed and finally the chain will be invalid. There are many more concepts around blockchain, such as mining, distributed peer-to-peer network protocols, consensus ledgers, cryptographic hashes, etc.

The "fingerprint" attribute represents the unique identifier of every block and is one of the core principles in the blockchain architecture. A commonly used algorithm for cryptography implementation on any digital data source is the well-known SHA256 hash, which was developed by the National Security Agency (NSA). SHA stands for secure hash algorithm and ' 256 ' is the number of bits it takes up in memory. There are five basic requirements that SHA256 satisfies:

1. One-Way decryption algorithm;

2. Deterministic (meaning that it will produce the same result each time the same input is used);

3. Fast computation;

4. Avalanche Effect;

5. Withstanding collisions [16].

The reason for using a hashing algorithm is because reverse engineering techniques are not practical. Thus, the importance of the use of the SHA256 algorithm lies on the fact that any attempt or effort to crack it is not realistically possible, due to its inherent characteristics (hashing is a one-way function).

Blockchain is also an immutable digital ledger, meaning that if anyone tries to tamper or corrupt the data of a specific block, thus changing the hash of this block, this will result in a cryptographic link disruption, due to the usage of different hash(es) between the linked blocks of the chain. Because of the change in a block in the chain, all the blocks after that will no longer be valid, which means that it will no longer be connected to the chain. Therefore, from the moment data have entered into the 
blockchain, they cannot be altered, as all the entries following the tampered block of the chain need to be altered as well. Because of this fundamental structure, it is practically impossible to change a single block in the chain, especially as more and more components are added. It has to be noted that any digital ledger is as reliable as the organization (blockchain on this occasion) that maintains it [17].

In case one or many blocks of the chain are maliciously changed, or a system error occurs in the process of data input, the cryptographic link will be broken and this will cause a data re-storage problem. This problem is solved through the deployment of distributed peer to peer (P2P) networks, which are one of the key components of blockchain technology and offer a significant enhancement of data storage technology compared to traditional centralized models. A P2P distributed network consists of a great number of computers where every device is interconnected (locally, wirelessly or by a cable); ideally the more they connect the better it is. The actual approach of how this type of distributed network is used can affect the whole blockchain scenario.

A P2P network stores and transfers data between the clients (or nodes) of the network without the need for a central point of storage (central server), so the data are less vulnerable to being hacked or lost. In a blockchain P2P network, the blocks of the chain can actually be copied across all the existing computers of the network (thousands or millions of computers) through the usage of the appropriate cryptographic key across all the peers. As time passes, the blockchain grows and the system becomes more and more complex. If someone attempts any malicious alteration on one or more blocks of the chain, they have to control, attack or manipulate $50 \%$ of the network's peers or more in order to break the blockchain, otherwise the other peers of the network will immediately realize the difference and will alert the whole network by sending a signal to replace the broken one. In this way, distributed P2P networks add an extra level of security on the existing cryptographic hash, which is a one-level security schema. In a consensus protocol, the more layers of security used, the stronger and safer the blockchain is made [18].

Every block that composes the blockchain contains at least four fields, namely:

1. The number of the block;

2. The stored data (or stored transactions);

3. The hash of the previous block;

4. The hash of the current block.

Another field in the block is called nonce (number used only once) and relates to the meaning of "mining". The hash of every block is dictated by four components, i.e., block number, the previous hash, the stored data, and nonce, and is generated by providing these as input to the SHA256 hashing algorithm. The block number, the previous hash, which is linked directly to contents of the previous block, as well as the stored data, cannot be changed because this would essentially mean that data tampering is attempted. Nonce provides additional control and flexibility that makes defining the correct hash value (one that meets certain requirements) possible, without the need to change any of the other components. In proof of work systems like blockchains, miners must find (by using brute force and significant computational power) a nonce value that, when plugged into the hashing algorithm, generates an output that meets specific requirements (e.g., a certain number of leading zeros).

Hence, mining can be perceived as the process of creating a new block for the blockchain and enriching it with a number of transactions. The mining difficulty indicator is the number that suggests the work intensity that a node's computer has to perform in order to create a new block. In the bitcoin blockchain, this is not a constant number but is automatically adjusted every 2016 blocks. Normally, the creation of this number of blocks should take exactly two weeks. In this period, if more blocks need to be created, the mining difficulty is increased, otherwise it is reduced. For the blockchain, this means that if more miners try to solve the cryptographic puzzle in a shorter timeframe, the system should increase the difficulty in order to maintain it. In an opposite situation (a lot of users may stop mining), the difficulty has to be reduced. In other words, the difficulty adjustment ensures that the 
mining process is performed for a specific amount of time, no matter how many users are mining or how fast the hardware is. One important disadvantage of this is that it tends to lead to centralization.

Decentralized applications (Dapps) on the blockchain are interfaces enabling people to connect and interact with many components of it. They are applications that can exist as decentralized programs that can run and can be stored on the peers' computers in the blockchain network rather than in a centralized server. The core visionary idea is to build a global super-computer in a distributed manner, which will be facilitated through a blockchain where everything (programs, transactions) will be recorded, tracked and stored in an immutable manner. Simultaneously, a copy of that blockchain application will reside with the clients.

\subsection{Ethereum and Smart Contracts}

Ethereum is a project platform that was created in 2013 by Vitalik Buterin. Essentially, the idea behind the Ethereum protocol is that all peers of a network are interconnected. Blockchain technology not only allows one to store transactional data but also to store and facilitate programs, as well as execute them, enabling any application to be decentralized [19]. The article of Vitalik Buterin, the founder of Ethereum, referred to the meaning of decentralization and presented the three different levels of the (de)centralization:

1. Logical (de)centralization;

2. Political (de)centralization;

3. Architectural (de)centralization [18].

Even today, many applications use the certification access mechanism which does not provide full visibility to the peers of the network. In order to address the aforementioned problem, in applications such as product supply chains, an environment that facilitates "smart contracts" is a promising approach. The term "smart contract" was firstly used by Nick Szabo in 1997. His main vision for smart contracts was the creation of a distributed ledger to store contracts. A contract is a set of rules or clauses that parties have agreed upon the governing relationship between them. Smart contracts are just like contracts in the real world, with the difference that they are completely digital. They are small script programs that are used and stored in blockchains, featuring a tamper-proof logic code into them. Smart contracts inherit some important attributes; they are immutable and distributed because of their storage inside the blockchain. Being immutable ensures that no one can tamper with the code of the contract, while being distributed secures the validation of smart contracts' output from everyone on the network. Smart contracts have been used in many types of blockchain applications such as in supply chains and in the health sector.

When a block (or transaction) is scanned and sourced through a completely digitized way, then the specific transaction is confirmed and the block is appended at the chain. After the execution of the contract, a certificate is issued, where a variety of information related to the blockchain transactions can be retrieved. Finally, every client in the network retains a copy of the smart contract and, as a result, each node has the following:

1. History of all smart contracts;

2. History of all transactions;

3. Current state of all smart contracts $[20,21]$.

Ethereum was specifically created and designed for smart contracts support. There are many examples of programming languages that allow software coding within the blockchain. A widely used tool for this purpose is Ethereum's Solidity programming language, a Turing-complete language. Solidity defines and determines a sequence of specific rules that dictate how a program operates and executes [19].

Table 1 provides an overview of the utilization of smart contracts in different agriculture traceability systems where blockchain technology is applied. As may be observed, the majority of current research 
results adopts the smart contract technology on the Ethereum blockchain in order to implement various types of transactions (such as transactions between farmers, suppliers and distributors).

Table 1. Survey or research works in the agriculture traceability systems—classification depending on the usage (or not) of smart contracts.

\begin{tabular}{|c|c|}
\hline Use of Smart Contracts & Literature Works \\
\hline Literature involving smart contracts in agriculture traceability systems & [22-58] \\
\hline $\begin{array}{l}\text { Literature involving blockchain technology but without the use of smart } \\
\text { contracts in agriculture traceability systems }\end{array}$ & {$[10,59-73]$} \\
\hline
\end{tabular}

\subsection{Consensus Methodologies}

A very important characteristic, not only for the blockchain technology, but also for any type of decentralized system, is byzantine fault tolerance. The byzantine generals' problem was conceived in 1982 as a logical dilemma that illustrates how a group of generals, who may have communication problems, will try to agree on the next move. If we apply the above definitions to the operation of blockchains, each general represents a network node, and all nodes need to reach a consensus on the current state of the system. This means that the majority of the participants within a distributed network have to agree and execute the same action in order to avoid failures. The byzantine generals' problem gave birth to the concept of byzantine fault tolerance. Byzantine fault tolerance is the property of a system to resist all the possible costs of failures derived from the byzantine generals' problem. In other words, a byzantine fault tolerant system is able to continue operating, even if some of the nodes fail to communicate or act in a malicious way. There are multiple ways of developing or building a byzantine fault tolerant blockchain system, and these are related to the different types of consensus algorithms/protocols [74,75].

A consensus algorithm can be defined as a mechanism through which a blockchain network reaches consensus. The consensus protocol for a blockchain has to solve two major challenges: the first one is to protect the network from attackers, and the second one is to tackle competing chains. There are multiple different types of consensus protocols:

1. Proof of work (PoW);

2. Proof of-stake (PoS);

3. Other protocols.

Proof of work (PoW) gives more rewards to people that own more and better equipment. As a result, the higher the hash rate of a user is, the higher the chance of creating the next block and receiving the mining award. In the end, the hash that every block has is the proof of work, which occurs by solving a cryptographic challenge puzzle. The term "mining pools" refers to the combination of hashing power and the distribution of the reward across every node in the winning pool. The use of mining pools renders the blockchain more centralized, as opposed to decentralized schemas.

In order to solve the above issue, an idea for a new blockchain consensus protocol, as an alternative to the proof of work protocol, was born. In 2011, a Bitcointalk forum user called QuantumMechanic proposed a new technique called 'proof of stake' (PoS). The differences between PoW and PoS are quite significant. PoS is more decentralized than PoW, it does not let every user to mine for new blocks, it is a lot less expensive compared to the PoW mining equipment requirements, and finally, encourages more people to set up a node, making the network more decentralized as well as more secure.

Besides these advantages, the PoS protocol entails additional risks when compared to PoW. Specifically, if a single or a group of miners can obtain $51 \%$ of the hashing power, they can effectively control and manipulate the blockchain. This attack is known as the $51 \%$ Attack. PoS makes this type of attack very impractical on specific cryptocurrency values, so it is actually less likely to occur with this type of consensus protocol, yet still remains an important risk. Another important risk issue is the way that PoS algorithms select the next validator. The process is random, and validators can typically be 
selected based on a combination of the lowest hash value and highest stakes, or based on how long their tokens have been staked for. Another problem of PoS is due to the possibility of selecting a user as the next validator, a role that may not come along its duties. An approach to solving this is by choosing a large number of backup validators [76].

\subsection{Public vs. Private Blockchain}

Adhering to the view for open access to everyone, many widely used blockchains (Bitcoin, Ethereum and Dash) are public networks. Public blockchain networks are characterized as permissionless, where anyone can join, read and/or write data, create smart contracts or even run a node within them, ensuring one hundred percent transparency as well as a high level of anonymity. Public networks are recommended for entities involved in crypto-economics. In contrast to public blockchain networks, private networks restrict either participant or validator access as a classic closed ecosystem where all peers are well defined and only preapproved entities can run nodes. Following a business-to-business approach, many companies use private blockchain networks in order to benefit from this technology without sacrificing their autonomy. Private blockchains, in contrast to public ones, typically use a type of consensus other than PoW, and might aim at keeping certain information private from the public. In summary, public blockchain networks enjoy freedom in decentralization, whereas private blockchain networks enjoy freedom in configurational flexibility [77]. Hybrid approaches also exist—-these are called permissioned blockchain systems. Different flavors and configurations of permissioned blockchain systems exist, but typically the consensus process is controlled by a pre-predefined list of participants, and users cannot participate without permission. Access to the full information of transactions on the blockchain might be restricted, depending on the user role.

Table 2 provides an overview of the types of blockchain used in different agriculture traceability systems. The majority of research works have used public blockchain types, in association with either Ethereum or Hyperledger, whereas a smaller percentage prefer the adoption of permissioned or fully private blockchains. Nonetheless, it is notable that many research studies attempt to make a more theoretic contribution and do not explicitly make reference to a specific blockchain implementation.

Table 2. Literature survey-use of different blockchain types and implementations in agriculture traceability systems.

\begin{tabular}{|c|c|c|}
\hline Public vs. Private Blockchain & Blockchain Implementation & Research Works \\
\hline \multirow{7}{*}{ Public } & Ethereum & {$[28-31,33,38,51,52,56,62,63]$} \\
\hline & Hyperledger Sawtooth & [54] \\
\hline & $\begin{array}{l}\text { Hyperledger (other than } \\
\text { Sawtooth) }\end{array}$ & {$[34,49]$} \\
\hline & Ethereum or Hyperledger & \\
\hline & Sawtooth & {$[43]$} \\
\hline & $\begin{array}{c}\text { Ethereum or Hyperledger (other } \\
\text { than Sawtooth) }\end{array}$ & [23] \\
\hline & Not specified & $\begin{array}{r}{[10,22,24,26,36,39,41,42,44,46,50} \\
55,59,60,64,65,67,69,70,72,73,78,79]\end{array}$ \\
\hline \multirow[b]{2}{*}{ Private } & Ethereum & {$[51]$} \\
\hline & $\begin{array}{l}\text { Ethereum or Hyperledger } \\
\text { Sawtooth }\end{array}$ & [23] \\
\hline \multirow{5}{*}{ Permissioned (Hybrid) } & Not specified & {$[26,32,47,50,66]$} \\
\hline & Ethereum & {$[27,40,48]$} \\
\hline & Hyperledger Sawtooth & {$[37,45,57,58]$} \\
\hline & Hyperledger Fabric & {$[35,53,68]$} \\
\hline & Not specified & {$[25,61,71]$} \\
\hline
\end{tabular}




\subsection{Traceability from Farm to Fork Using Blockchains}

Blockchain technology provides the ability to create a smarter and more secure supply chain, offering a clear and solid audit trail of the tracked products in real-time. Traceability information regarding product origin and possible allergens or added substances, which has to be stored in a secure and immutable manner, can be established and shared through a collaborative blockchain network between farmers, manufacturers and distributors. Such blockchain networks can exist at global scale (as done today in the case of many crypto-currencies), spanning across multiple countries and organizations, establishing a trustworthy information flow among stakeholders with specific agreements when it comes to data exchange. According to Robert Sinfield, Vice President of Product for Sage Business Cloud X3 [80]: “On the visibility side, blockchain ERP systems could enable everyone involved to track the product's journey from the manufacturing floor to the retailer's shelf, without having to worry about records being lost or tampered with ... Nowhere is this more prevalent than in the food and drink space, where blockchain will provide transparency and product provenance that is validated from farm to fork".

\section{Literature Review and Related Work}

The previous sections of this paper made a brief yet comprehensive overview analysis of the concepts and terms of traceability, DLT and blockchains. Blockchains are an emerging technology, and despite the fact that there are numerous publications and case studies in the field, a relatively limited number of them focus on and specialize in the agriculture sector and the food supply chain. This section performs a literature review of relevant experimental applications proposed for traceability in the management of agriculture supply chains.

\subsection{Early Solutions for Traceability Systems (2009-2012)}

The early electronic traceability systems were centralized solutions based on databases and data import conducted either in a manual or semiautomatic way. Gandino et al. (2009) propose a framework consisting of RFID tags attached on products in a fruit warehouse [81]. The proposed system is a semiautomatic RFID-based traceability platform which was designed in order to test and evaluate the effectiveness and the potential improvement of traceability using automation improvements. Specifically, the experimental system uses RFID tags for reading products' attributes, RFID readers in order to obtain the data from the tags, personal digital assistant (PDA) devices for the personnel, so they could read the RFID readers, and a central computing system with a central database where the obtained data from the RFID tags on products are stored. This specific case study revealed that the application of RFID technology on agriculture traceability can provide many advantages and improvements, such as shorter time for data management and analysis.

Another traceability system which is based on a central computing system is proposed by C. Cheng et al. (2012) [82]. The proposed framework consists of printed tags with traceability codes for every product, XML (extensible markup language) files containing all the required data regarding the traceable resource units (TRUs), and a central database where all the data are stored and made available for the stakeholders. The system is both efficient and precise when it comes to limited data streams. However, as the data volume increases, the database is heavily loaded and thus additional computational power is needed.

Corrado Costa et al. made a review on RFID and agri-food supply chain traceability in [9], in which the advantages and the possible challenges regarding the application of RFID technology in the food supply chain are elaboratively presented. Furthermore, a conceptual idea of a cloud-based agriculture traceability system is formulated as a future work proposal. Since then and while the blockchain technology has been gaining more and more ground in data science, the first ideas and proposals for cloud traceability systems using DLTs started to formulate. 
The above research efforts represent the very first steps of automated electronic traceability systems (including those based on DLT). Those systems provided or improved the means for the collection of traceability information regarding agri-products by using communication technologies such as RFID and wireless sensor networks.

\subsection{Distributed Ledger Solutions (2016-Today)}

Following the first electronic traceability systems for agriculture products described above, and as the blockchain technology was maturing and becoming more mainstream in other application domains, more and more research efforts emerged, concentrating on the use of DTL for agriculture traceability systems. Feng Tian (2016) proposes a revolutionary idea about a system based on RFID and blockchain technology for Chinese agri-food markets with the aim of enhancing their food safety and quality, as well as reducing losses during logistic processes [10]. Tian's work is one of the most cited in the literature regarding blockchain applications in the agriculture sector. According to Tian, RFID and blockchain technology are used to guarantee food safety and quality throughout the entire supply chain. The paper examines two types of agriculture products: (i) fresh fruits and vegetables, and (ii) meat, such as pork, chicken and beef. The proposed network utilizes the features of blockchains so that all stakeholders can have access to every transaction and information regarding a specific product. The basic aim of Tian is to cover the whole process of data collection and information management, for every transaction between the stakeholders in the agriculture supply chain. The entire system provides monitoring, tracking and tracing of the quality of agri-food and can be characterized as a "farm to fork" solution. Tian's solution presents both advantages and challenges (considering societal, financial and technical aspects) compared to centralized solutions.

Henry M. Kim (2016) proposes an ontology-driven blockchain traceability system. The main idea of Kim is to translate and define some crucial traceability components as different ontologies. Similarly with Feng Tian [10], Kim utilizes blockchain technology in order to propose a traceability system with various ontologies, where each one could accomplish and be part of certain transactions. Alongside blockchain technology and ontologies, Kim proposes the use of smart contracts. The platform used in this paper is Ethereum, programmed with the Solidity programming language [83].

Feng Tian et al. continue their research in 2017 and propose a blockchain solution for agriculture traceability in order to ensure that the HACCP principles and requirements are addressed during the production, transportation and preservation of a product [36]. Moreover, Daniel Tse et al. (2017) focus on the increasingly serious problem of food safety in China and proposes a blockchain solution for the agriculture supply chain, based on information and transaction security between all the involved parties. In this paper, a PEST (political, economic, social, technological) environment analysis is presented in order to define the challenges and the opportunities of the DLT solution [84]. Francesco Marinello et al. also propose a blockchain solution focusing on the animal products supply chain in Italy [59]. They conduct an analysis of the animal products supply chain in order to identify the discrete steps of tracking and tracing a product. Additionally, in the context of different scenarios, they study the way in which the number of involved parties at each supply chain step could affect the complexity level of information flow. In this direction, Visser et al. propose a blockchain framework which can help to improve tuna traceability and stop illegal and unsustainable fishing practices [67]. Considering the safety of agriculture products, Kumar et al. [41] propose a rice supply chain system that uses blockchain technology to assure the safety of rice during its flow through the supply chain.

The growing research interest in blockchains and traceability in agriculture gained further ground in 2018 when many research efforts on this issue were published. Leng et al. discuss a dual chain solution for the agriculture supply chain. Leng studied the complexity and the problems of China's agriculture sector, concluding that poor organization is a very limiting issue [22]. Considering this, the proposed dual blockchain system consists of a user information chain and a transaction chain. The user information chain is used to record and store the user information of the agricultural business 
enterprises in the public service platform, while the transaction chain is used to record and store all transaction data. Results of the conducted trials of the proposed framework show that the double-chain solution not only ensures the transparency and security of transaction information and the privacy of enterprise information, but can also significantly improve the credibility of the public service platform and the overall system efficiency.

Maria Elena Latino et al. propose another interesting idea regarding the agriculture supply chain and the use of Industry 4.0 principles [85]. Latino et al. refer to the idea of food democracy, according to which consumers are considered as citizens and the food not as a good but as a civil right. The authors advertise the idea of voluntary traceability and combine it with Industry 4.0 technologies. Their proposal concentrates on a framework consisting of a big data platform for handling collected data. This enables maintaining data paternity with respect to the supply chain operator and traceability towards the product. The significance of the voluntary traceability is highlighted, focusing on the volume and the quality of the data collected for each product, as well as the need for a big data platform to handle them.

Caro et al. propose an integrated solution of a blockchain platform named AgriBlockIoT in the agriculture supply chain [23]. AgriBlockIoT is a fully distributed system which uses the blockchain technology in combination with IoT devices in order to collect and distribute traceability data. The proposed solution was tested with two different blockchain platforms, namely Ethereum and Hyperledger Sawtooth. Trial results showed that Ethereum performed considerably better compared to Hyperledger Sawtooth, in terms of latency, CPU and network usage. AgriBlockIoT enables the integration of IoT and blockchain technologies, creating transparent, fault-tolerant, immutable and auditable records which can be used for an agri-food traceability system. Furthermore, Lin et al. propose another system based on blockchain and IoT technologies for the agriculture supply chain [60]. Specifically, the authors try to combine the technologies of blockchain, IoT, low-power wide-area network (LPWAN) and existing enterprise resource planning (ERP). The proposed platform, which aims to solve food safety and trust issues of the traditional agri-food supply chain, involves all parties within a typical agriculture supply chain ecosystem.

In 2018, many more articles on the application of blockchain technology into the agriculture supply chain were published. A number of researchers $([5,24-28,33,34,46,52,65,86])$ propose similar solutions regarding the integration of blockchain in the agriculture supply chain with small scale differentiations. Mao et al. [27,52] propose the FTSCON (Food Trading System with Consortium blockchain), an automated merchant transaction mechanism for the agri-food supply chain. FTSCON improves transaction security and privacy protection by using smart contracts and a consortium (private) blockchain which is more efficient than a public one in terms of financial cost and computational power. Mark Kim et al. [28] introduce the Harvest Network, a blueprint to build a "farm to fork" food traceability application, where they combine the Ethereum blockchain and IoT devices with GS1 message standards. The Harvest Network introduces the idea of tokenized smart contracts, in which the contract is not subject to global consensus and does not have to be validated by the entire network, but instead is only be processed by dynamically sized node clusters, thus improving efficiency.

Arsyad et al. [72] propose a framework that uses a two-way factor verification blockchain solution and watermarks images and documents using the discrete wavelet transform method. Hayati et al. [54] present the FoodTrail platform, which uses a four-layer architecture in conjunction with smart contracts as a way to conduct transactions through the whole supply chain. In the same year, the World Wildlife Fund (WWF) creates a project called "Bait-to-plate" focused on the traceability of tuna in New Zealand throughout the whole supply chain. WWF's project adopts RFID technologies for fish tagging and an Ethereum-based blockchain [63].

The research interest in blockchains in the agriculture sector continues its upward trend as more and more researchers, companies and scientists expand the existing knowledge base in 2019. Koirala et al. propose a consortium blockchain solution where a central authority (CA) defines the stakeholders of the network as well as the certifications which allow them to participate in it. The authors present 
a detailed description of their proposed model and algorithms, as well as the proof of concept (PoC) method that is used to validate the smart contract transactions [87]. Following the same concept, Baralla et al. [29] propose a framework using a consortium, Ethereum-based blockchain and smart contracts for the food supply chain in the Sardinia region. Baralla et al. highlight the positive aspects of using this approach in order to promote smart tourism and protect the authenticity of Sardinian local products. Another interesting publication considering consortium blockchains and smart contracts is formulated in the publication of Lin et al. [30]. More specifically, Lin et al. propose a system which combines blockchain, smart contracts and electronic product code information services (EPCIS). In order to make the blockchain more efficient, they promote the idea of reducing the data stored on the blockchain, by using an on-chain/off-chain approach where the on-chain data are those required to track and trace a product, and the off-chain data are enterprise data, most of which are confidential. Through this approach, the blockchain avoids being heavy-loaded and the sensitive data of enterprises are protected. In this direction, Umamaheswari et al. present a platform that stores data from crop sensors in an Ethereum-based blockchain, which facilitates smart contracts for buying or selling crops in India [43].

A state-of-the-art analytical approach regarding the blockchain and smart contracts implementation in the agri-food supply chain is described by Khaled Salah et al. in their publication [31]. This paper demonstrates how blockchain and Ethereum smart contracts can efficiently trace and track, as well as enable, a fully functional integration of stakeholders' transactions in the agriculture supply chain. The authors propose a series of algorithms regarding smart contract validation and execution as part of the implementation framework. The proposed solution is applied for tracing and tracking in the soybean supply chain, but could be generalized to provide trusted and decentralized traceability to other types of crops and products in the agriculture supply chain. Scuderi et al. study the application of blockchains in protected designation of origin (PDO) and protected geographical indication (PGI) products in the European Union. Their study makes it possible for consumers to verify the origin of the raw materials, as well as obtain information on the production process, the process controls, the packaging operations, and the distribution and certification related to the product, in order to detect possible adulteration [70].

Greydt and Fischer present an analysis about the necessity of blockchains in the agriculture sector, also presenting the three generations of blockchains (Blockchain 1.0, Blockchain 2.0 and Blockchain 3.0) and their attributes [88]. Greydt and Fischer also refer to the evolution of blockchain technology in which data will no longer be stored in blocks but as a directed acyclic graph (DAG). Through this approach, it is estimated that data can be processed much faster, which could address the scalability problem of throughput, latency and capacity regarding the execution of transactions. In the same context, Kamble et al. conduct a thorough analysis in order to model a blockchain enabled traceability system in the agriculture supply chain. Through interpretive structural modeling (ISM) and decision-making trial and evaluation laboratory (DEMATEL) methodologies, Kamble et al. establish hierarchical levels and relationships between the involved actors in the supply chain [32]. The study identifies thirteen enablers for implementing blockchain technology in agriculture supply chains, such as anonymity and privacy, immutability, smart contracts, secured and shared database, traceability, transparency, and others; the enablers were validated by agriculture supply chain experts. The authors also highlight the need to further involve experts from various countries in order to ensure consideration of diversity factors and relevant indicators.

Another interesting idea in this direction, presented by Mondal et al., describes a blockchain-IoT based system which utilizes the proof of object (PoO) concept as an alternative to the blockchain's proof of work (PoW) and proof of stake (PoS). PoO is a validation method where the owner of the object is obliged to prove the possession. As long as the other stakeholders validate this claim, a consensus is reached and a new block is added in the blockchain. The authors follow an analytical approach and present trial results for the proposed model regarding the implementation of the consensus algorithm, the security issues and the involved sensor technology [61]. 
In 2019, important similar studies focusing on the application of blockchains in agriculture traceability systems appear. Liao et al. in [62] propose a safety management system for tea quality, based on a public Ethereum blockchain. Moreover, Wang and Liu in [49] present a general architectural concept for blockchain implementation in agricultural product traceability systems. Kakkar et al. [73] describe a blockchain application in a rice supply chain, with the aim of changing the way in which the food corporation in India manages the supply chain, as well as enabling significant wastage reduction. Furthermore, several studies present concepts and implementations of blockchain-based traceability systems regarding different agricultural products, such as chicken [69], bananas, fish and meat [51], maize [56], and pork [79].

The upward trend of research interest regarding blockchain and agriculture is depicted in Figure 2 below, which demonstrates the trend of Scopus documents with the search term TITLE-ABS-KEY(agriculture AND blockchain) from 2017 to 2019.

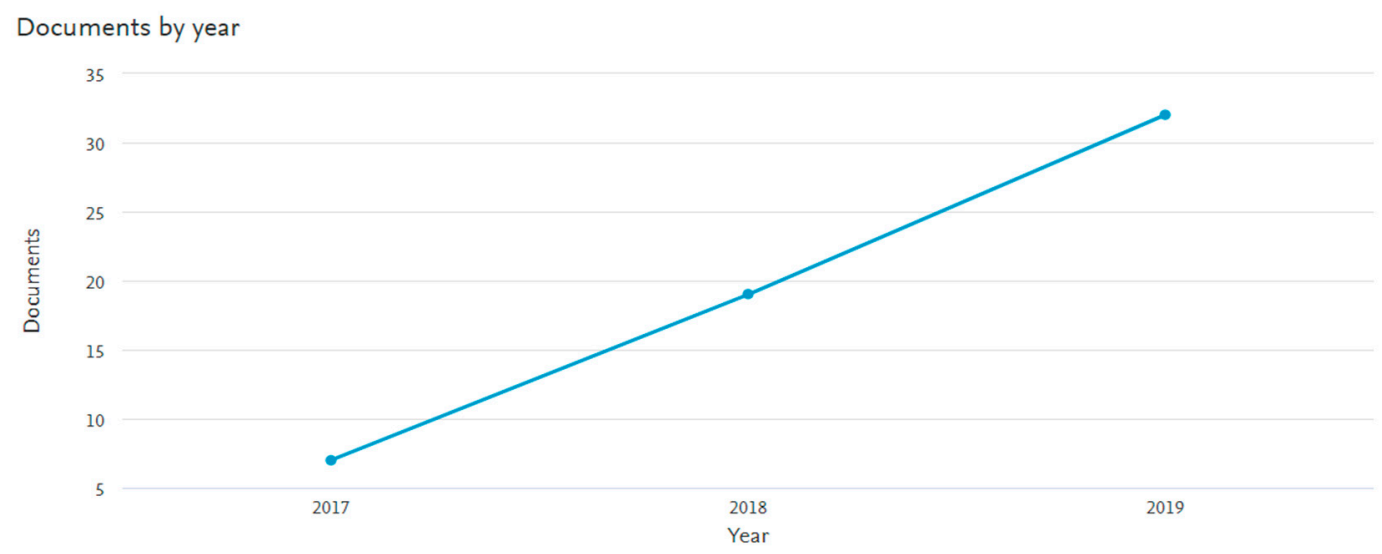

Figure 2. Scopus number of document for TITLE-ABS-KEY(agriculture AND blockchain) from 2017 to 2019 .

The research interest in this specific domain continues to grow also in 2020. Zhang et al. [42] present a conceptual architecture to store high-throughput crop breeding data, in an efficient and safe manner, by using blockchain technology in a breeding information management system. Furthermore, Sabir et al. focus on the potential arising from DLT in the smart farming initiative, due to more transparent, safe and efficient data sharing [44]. Gunasekera et al. [55] study the effect transaction costs reduction due to the use of a blockchain as a DLT in grain trading in Australia.

Table 3 depicts the classification of relevant research works regarding the use of blockchain technology in agriculture traceability systems, according to the agriculture sector and product. The majority of research results fall into the agri-food sector, followed by general solutions for the agriculture supply chain and the livestock sector. It is notable that, especially in the agri-food sector, several research works do not make explicit reference to a specific type of product. 
Table 3. Applications of blockchain technology for traceability across different agriculture sectors and products.

\begin{tabular}{|c|c|c|}
\hline Agriculture Sector & Agriculture Product & Research Works \\
\hline \multirow{9}{*}{ General agriculture supply chain } & Farming products & {$[23,26,32,40,68]$} \\
\hline & Crops & {$[42,43]$} \\
\hline & Not specified & {$[22,44,49,65]$} \\
\hline & Bananas & [51] \\
\hline & Cacao and chocolate & {$[71,72]$} \\
\hline & Eggs & [37] \\
\hline & Fresh fruit and vegetables & [10] \\
\hline & Grains & {$[34,55]$} \\
\hline & Grapes (table grapes) & {$[53]$} \\
\hline \multirow[t]{7}{*}{ Agri-food } & Maize & [56] \\
\hline & Pasta (Spaghetti) & [54] \\
\hline & Peppers & [57] \\
\hline & Rice & {$[41,73]$} \\
\hline & Tea & [62] \\
\hline & Wheat, corn or soybean & {$[31,52]$} \\
\hline & Not specified & {$[24,27-30,36,45,47,48,60,61,70,78]$} \\
\hline \multirow[t]{2}{*}{ Beekeeping } & Honey products & {$[46]$} \\
\hline & Salmon (wild salmon) & [38] \\
\hline \multirow[t]{4}{*}{ Fishing } & Tuna & {$[63,67]$} \\
\hline & Fish (general) & {$[35,50,51]$} \\
\hline & Chicken & {$[25,58,69]$} \\
\hline & Halal meat & [39] \\
\hline \multirow[t]{3}{*}{ Livestock } & Pork & [79] \\
\hline & Turkey & [64] \\
\hline & Meat (misc.) & {$[10,51,59]$} \\
\hline Timbering & & [33] \\
\hline Vinification & & {$[29,66]$} \\
\hline
\end{tabular}

\section{Commercial Solutions}

While scientific research regarding blockchains and traceability in agriculture supply chains is growing rapidly, the same is not reflected in the availability of commercial applications. One of the most celebrated relevant applications is IBM Food Trust. IBM Food Trust was tested with the collaboration of Walmart in order to trace the provenance of mango and Chinese pork products. It is built on the Hyperledger Fabric platform where all data are stored on blockchain ledgers and are accessible throughout the entire supply chain from producer to consumer. An indicative advertised outcome of IBM Food Trust is that the process of identifying a mango's provenance could take seven days of tracking without the Food Trust system, whereas with the Food Trust, it took approximately around $2.2 \mathrm{~s}[89,90]$.

Provenance is another company that promotes a blockchain-based traceability system for fish products [91]. Through the company's proposed application, every fish product is tagged uniquely. This unique ID contains all the information about the fish, its packaging, transportation and storage conditions. The aim of Provenance is to reduce the double certification costs and provide trustworthy information to consumers by using an immutable distributed ledger system. Provenance utilizes a public blockchain where all the stakeholders are equal, and the information security is high.

Following the leading steps of IBM's Food Trust and Provenance, nowadays, more and more startup companies emerge in the territory of blockchain-supported agri-food traceability. For example, the AgriOpenData product aims to guarantee, in a transparent, secure and public manner, the traceability of the whole agri-food chain, as well as the processing of agricultural products, especially BIO (biological- in Europe often used as synonym to organic) and DOCG (denomination of controlled and guaranteed origin) products, allowing them to certify the quality and their digital 
identities though a blockchain implementation [92]. Some of the companies that conduct business in this context, and with very similar functionalities and characteristics, are shown in Table 4.

Table 4. Companies and websites which promote blockchain solutions for agriculture supply chains.

\begin{tabular}{cc}
\hline Company/Commercial Product & Website \\
\hline agrichain & https://agrichain.com \\
agridigital & https://www.agridigital.io \\
agriledger & http://www.agriledger.io/about/ \\
arc-net & https://arc-net.io \\
Bühler Smart Supply Chain & https://digital.buhlergroup.com/smartsupplychain/ \\
Demeter.life & https://demeter.life \\
DOWNSTREAM & https://www.down-stream.io \\
Fishcoin & https://fishcoin.co \\
Honeysuckle White & https://www.honeysucklewhite.com \\
Ripe.io & https://www.ripe.io \\
TE-FOOD & https://tefoodint.com \\
\hline
\end{tabular}

\section{Discussion-Challenges and Future Work}

\subsection{Blockchain Implementation Challenges}

As can be seen from the above, blockchain technology is gaining more and more ground in the supply chains sector. Blockchains and DLT may solve the vast majority of existing issues, although they are not a panacea, especially considering the fact that the agriculture supply chain is very sensitive to criticism and, in many cases, can operate under overwhelming pressure. In parallel, the use of blockchain technologies must also take into consideration various societal, technical and financial factors.

Regarding the societal impact of blockchain adoption, T. Burke in [12] notices that the agriculture sector has its own unwritten rules and fragile relationships that should not be disturbed. In a more subtle wording, the agri-food supply chain is very complex and reluctant to a blockchain adoption for the following reasons:

1. Technological knowledge of many stakeholders is of low level;

2. Products throughout the chain undergo many transformations;

3. The roles and business of the large number of stakeholders involved are extremely heterogeneous;

4. The food supply chain is distributed to large geographical areas, even in different continents across the globe, a fact that poses significant interoperability and deployment obstacles.

What is more, issues related to data management, particularly data ownership and data retention within the blockchain, are also important and have to be carefully considered [93].

From a technical perspective, blockchains may be immutable ledgers, but the accuracy of data that are inserted by the sensors or by persons cannot be guaranteed. Thus, for example, if a sensor malfunctions, then the information across the blockchain is not accurate [86]. In general, there are difficulties in the monitoring, integration and evaluation of certain categories of data within the agricultural supply chain. For instance, environmental data are hard to retrieve and assess using objective methods [88].

Another important technical issue is the cooperation and seamless integration of blockchains with legacy traceability systems, preserving in parallel blockchains' distributed nature as a DLT. Most of the works surveyed and presented in Sections 3 and 4 are research efforts that have been tested in laboratory environments, with a limited number of users and transactions. The scalability of such traceability systems remains a challenge in the context of larger implementations and deployments [93].

The selection of the proper type (public, private, permissioned) and implementation of blockchain is also an associated issue. As observed from Table 2 in Section 3.4, a large number of research efforts do 
not concretely specify the blockchain implementation, still concentrating on more conceptual designs. Moreover, a large number of research works (Section 3.4) use public blockchains, and the same applies to some of the commercial products identified in Section 5. However, the trade-offs of this choice in terms of performance are not always sufficiently discussed; this is because, in agri-food supply chains that involve several actors, the completion of a transaction using a public blockchain can take a considerable amount of time and computing power. In this respect, the selection of the type of blockchain to be used should be done with care, as it might affect the traceability system's performance.

From a financial point of view, blockchains may increase the transparency of the food supply chain as well as consumer trust, but its usage comes with an enormous energy and financial cost [61]. It is mandatory for companies to invest a lot of money, as well as time, to train all the involved personnel and also to obtain the required equipment. On the other hand, such investments in time and money may lead to high traceability costs which may exceed the product's own cost, both in short and long term, thus a cost-benefit analysis is essential.

A very interesting study considering the challenges of blockchain applications in the agriculture supply chain was conducted Kay Behnke and Marijin Jansen [94]. This study identifies 18 boundary conditions for food traceability, 5 of which directly apply to blockchain technology. According to the authors, a significant number of these are related to regulatory requirements, the internal supply chain and production processes, which require significant organizational changes in order to support the full benefits of traceability. In the same context, Kamilaris et al. make an analysis of the blockchain's impact on the agriculture supply chain, and discuss the overall implications and challenges [95]. The main challenges highlighted include the accessibility to blockchain technology, governance and sustainability, policy and regulation rules, technical challenges and design decisions that must be taken, as well as the bridging of the digital gap between developed and developing countries in order to achieve successful adoption of such emerging technologies. Kamilaris et al. also refer to the important role of governments as a leading example by fostering the digitalization of public administration. They also argue that investments in technology and education must be carried out in order to further produce and demonstrate evidence considering the benefits of the new blockchain technology.

\subsection{Future Prospects}

The previous sub-section provided an overview of the main identified blockchain challenges regarding the technology's potential integration in the agriculture sector. Taking into account these challenges, future research efforts should focus on how to tackle and overcome them. Moreover, researchers and developers should concentrate on how the blockchain can be made even safer, and in parallel, more efficient. Blockchains can be considered as a mature approach regarding financial technology (FinTech) and cryptocurrencies, but as far as the supply chain is concerned, it is still in a nascent stage. Thus, besides the technical problems and challenges, future discussion must also be aimed at the necessary legislation and policies for the integration of blockchain technologies in a fragile and sensitive sector as the agriculture. Furthermore, fast developing technologies involving artificial intelligence (AI) in combination with DLT could lead to the establishment of the smart agriculture paradigm, where all the different services, components and stakeholders would be interconnected. Smart agriculture, apart from the enhancement of traceability systems, could also provide benefits towards more efficient production, by leveraging big data and machine learning algorithms. Thus, scientific research should be steered towards a more practical approach for creating pilot applications and platforms in the next years, as well as for expanding the boundaries of DLT by combing them with big data, $\mathrm{AI}$ and machine learning techniques, in order to create a smarter, safer, and well protected agriculture sector.

\section{Conclusions}

The goal of this study was to conduct a thorough research in the literature concerning traceability techniques and blockchain technologies and their combination in the agriculture sector. Traceability is 
a field that has been well studied throughout the years. Several regulations, directives and laws around the world regarding the traceability of agri-food products have been established. On the other hand, although blockchain technology has been a subject of extensive research over the last decade, it is only in the last few years that some research studies on the implementation of blockchain in agriculture traceability systems have made their appearance. At the same time, an increasing trend on startups and pilot applications is observed.

Research conducted in the context of this paper showed that using blockchains can advantageously help to achieve traceability by irreversibly and immutably storing data. Blockchain technology creates a unique level of credibility that contributes to a more sustainable food industry. Although assuring food traceability with blockchain technology looks promising, certain limits remain to be considered and addressed, including regulations, relationships between stakeholders, data ownership, scalability, etc. In order to better understand the technology and, possibly, generate new implementations, researchers and developers would benefit from the development of a universal evaluation model.

To conclude, for the implementation of a new system to be characterized as successful, this system should empower the following: (i) reduce costs, (ii) reduce risk, (iii) save time, and (iv) increase trust and transparency. Stakeholders are willing to adopt a new way of working only when they are convinced that the proposed method is user-friendly, increases productivity and brings added value. Taking the above into account, it is clear that the consolidation of new technologies in the traditional sector of agriculture is an enormous challenge which should be carried out step by step, and only by efficiently engaging the directly affected stakeholders across the supply chain.

Author Contributions: K.D. was the paper initiator. He identified the potential of using blockchain technologies in the supply chain of the agri-food sector and conducted research on the prospective and limitations of relevant traceability systems. N.P. worked on studying blockchain-related issues, including their different implementation approaches, the foreseen consensus methodologies, and the application of smart contracts. T.A. researched the applicability of blockchain solutions in the agri-food domain, especially the implementation of different blockchain types in agriculture traceability systems. E.A. performed research on applications of blockchain technology for traceability across different agriculture sectors and products. It is noted that all authors closely cooperated in order to achieve suitable information flow and harmonization across the entire paper. All authors have read and agreed to the published version of the manuscript.

Funding: This research was funded by the Computer Networks Laboratory of the Institute of Communication and Computer Systems, Greece.

Conflicts of Interest: The authors declare no conflict of interest.

\section{References}

1. Almeida, O.X.B.; Rodriguez, M.C.; Samaniego, T.; Gomez, E.C.F.; Cabezas-Cabezas, R.; Bazan, W. Blockchain in agriculture: A systematic literature review. In Proceedings of the Technologies and Innovation, Guayaquil, Ecuador, 6-9 November 2018; Valencia-García, R., Alcaraz-Mármol, G., Del Cioppo-Morstadt, J., Vera-Lucio, N., Eds.; Springer International Publishing: Cham, Switzerland, 2018; pp. 44-56.

2. Traceability in Food and Agricultural Products. Available online: http://www.intracen.org/uploadedFiles/ intracenorg/Content/Exporters/Exporting_Better/Quality_Management/Redesign/EQM\%20Bulletin\%20912015_Traceability_FINAL\%2014Oct15_web.pdf (accessed on 19 October 2019).

3. ISO Technical Committee. Traceability in the Feed and Food Chain-General Principles and Basic Requirements for System Design and Implementation; ISO 22005:2007; ISO Technical Committee: Geneva, Switzerland, 2016.

4. European Parliament and of the Council. 28 January 2002 laying down the general principles and requirements of food law, establishing the European Food Safety Authority and laying down procedures in matters of food safety. In Official Journal of the European Union 31; European Parliament Regulation (EC) No 178/2002; European Parliament and of the Council: Brussels, Belgium, 2002; pp. 1-24.

5. Corallo, A.; Paiano, R.; Guido, A.L.; Pandurino, A.; Latino, M.E.; Menegoli, M. Intelligent Monitoring Internet of Things Based System for Agri-food Value Chain Traceability and Transparency: A Framework Proposed. In Proceedings of the 2018 IEEE Workshop on Environmental, Energy, and Structural Monitoring Systems (EESMS), Salerno, Italy, 21-22 June 2018; pp. 1-6. 
6. Aung, M.M.; Chang, Y.S. Traceability in a food supply chain: Safety and quality perspectives. Food Control 2014, 39, 172-184. [CrossRef]

7. Verzijl, D.; Rouwmaat, E.; Dervojeda, K.; PwC, N.; Probst, L.; Frideres, L.; PwC, L. Traceability across the value chain-standards, processes and traceability. In Business Innovation Observatory; European Union: Brussels, Belgium, 2015.

8. Pappa, I.C.; Iliopoulos, C.; Massouras, T. What determines the acceptance and use of electronic traceability systems in agri-food supply chains? J. Rural Stud. 2018, 58, 123-135. [CrossRef]

9. Costa, C.; Antonucci, F.; Pallottino, F.; Aguzzi, J.; Sarriá, D.; Menesatti, P. A review on agri-food supply chain traceability by means of RFID technology. Food Bioprocess Technol. 2012, 6, 353-366. [CrossRef]

10. Feng, T. An agri-food supply chain traceability system for china based on RFID \& Blockchain Technology. In Proceedings of the 2016 13th International Conference on Service Systems and Service Management (ICSSSM), Kunming, China, 24-26 June 2016; pp. 1-6.

11. Shi, X.; An, X.; Zhao, Q.; Liu, H.; Xia, L.; Sun, X.; Guo, Y. State-of-the-art internet of things in protected agriculture. Sensors 2019, 19, 1833. [CrossRef] [PubMed]

12. Burke, T. Blockchain in food traceability. Food Traceability 2019, 133-143. [CrossRef]

13. Haber, S.; Stornetta, W.S. How to time-stamp a digital document. J. Cryptol. 1991, 3, 99-111. [CrossRef]

14. Nakamoto, S. Bitcoin: A Peer-to-Peer Electronic Cash System. Cryptography Mailing List. 2008. Available online: https://metzdowd.com (accessed on 20 May 2020).

15. Raikwar, M.; Gligoroski, D.; Kralevska, K. SoK of used cryptography in blockchain. IEEE Access 2019, 7, 148550-148575. [CrossRef]

16. Penard, W.; van Werkhoven, T. On the secure hash algorithm family. In Cryptography in Context; Wiley: Hoboken, NJ, USA, 2008; pp. 1-18.

17. Berg, C.; Davidson, S.; Potts, J. The Blockchain Economy: A Beginner's Guide to Institutional Cryptoeconomics. Available online: https://medium.com/cryptoeconomics-australia/the-blockchain-economy-a-beginnersguide-to-institutional-cryptoeconomics-64bf2f2beec4 (accessed on 21 October 2019).

18. Buterin, V. The Meaning of Decentralization. Available online: https://medium.com/@VitalikButerin/themeaning-of-decentralization-a0c92b76a274 (accessed on 18 October 2019).

19. What is Ethereum? The Ultimate Beginners' Guide. Available online: https://coincentral.com/what-isethereum-the-ultimate-beginners-guide/ (accessed on 21 October 2019).

20. Project Provenance Ltd Blockchain: The Solution for Transparency in Product Supply Chains. Available online: https://www.provenance.org/whitepaper (accessed on 22 October 2019).

21. Jabbari, A.; Kaminsky, P. Blockchain and Supply Chain Management. In Proceedings of the College Industry Council on Material Handling Education, Phoenix, AZ, USA, 27 September 2018.

22. Leng, K.; Bi, Y.; Jing, L.; Fu, H.-C.; Van Nieuwenhuyse, I. Research on agricultural supply chain system with double chain architecture based on blockchain technology. Future Gener. Comput. Syst. 2018, 86, 641-649. [CrossRef]

23. Caro, M.P.; Ali, M.S.; Vecchio, M.; Giaffreda, R. Blockchain-based traceability in Agri-Food supply chain management: A practical implementation. In Proceedings of the 2018 IoT Vertical and Topical Summit on Agriculture-Tuscany (IOT Tuscany), Tuscany, Italy, 8-9 May 2018; pp. 1-4.

24. Casado-Vara, R.; Prieto, J.; De La Prieta, F.; Rodríguez, J.M.C. How blockchain improves the supply chain: Case study alimentary supply chain. Procedia Comput. Sci. 2018, 134, 393-398. [CrossRef]

25. Hong, W.; Cai, Y.; Yu, Z.; Yu, X. An agri-product traceability system based on IoT and blockchain technology. In Proceedings of the 2018 1st IEEE International Conference on Hot Information-Centric Networking (HotICN), Shenzhen, China, 17-19 August 2018; pp. 254-255.

26. Schmidhuber, J.T.M. Emerging Opportunities for the Application of Blockchain in the Agri-food Industry; Food and Agriculture Organization of the United Nations and International Centre for Trade and Sustainable Development (ICTSD): Geneva, Switzerland, 2018.

27. Mao, D.; Hao, Z.; Wang, F.; Li, H. Novel automatic food trading system using consortium blockchain. Arab. J. Sci. Eng. 2018, 44, 3439-3455. [CrossRef]

28. Kim, M.; Hilton, B.; Burks, Z.; Reyes, J. Integrating blockchain, smart contract-tokens, and IoT to design a food traceability solution. In Proceedings of the 2018 IEEE 9th Annual Information Technology, Electronics and Mobile Communication Conference (IEMCON), Vancouver, BC, Canada, 1-3 November 2018; pp. 335-340. 
29. Baralla, G.; Ibba, S.; Marchesi, M.; Tonelli, R.; Missineo, S. A blockchain based system to ensure transparency and reliability in food supply chain. In Proceedings of the Euro-Par 2018: Parallel Processing Workshops, Turin, Italy, 27-28 August 2018; Mencagli, G., Heras, D., Cardellini, V., Casalicchio, E., Jeannot, E., Eds.; Springer International Publishing: Cham, Switzerland, 2019; pp. 379-391.

30. Lin, Q.; Wang, H.; Pei, X.; Wang, J. Food safety traceability system based on blockchain and EPCIS. IEEE AccesS 2019, 7, 20698-20707. [CrossRef]

31. Salah, K.; Nizamuddin, N.; Jayaraman, R.; Omar, M. Blockchain-based soybean traceability in agricultural supply chain. IEEE Access 2019, 7, 73295-73305. [CrossRef]

32. Kamble, S.S.; Gunasekaran, A.; Sharma, R. Modeling the blockchain enabled traceability in agriculture supply chain. Int. J. Inf. Manag. 2020, 52, 101967. [CrossRef]

33. Figorilli, S.; Antonucci, F.; Costa, C.; Pallottino, F.; Raso, L.; Castiglione, M.; Pinci, E.; Del Vecchio, D.; Colle, G.; Proto, A.R.; et al. A blockchain implementation prototype for the electronic open source traceability of wood along the whole supply chain. Sensors 2018, 18,3133. [CrossRef]

34. Lucena, P.; Binotto, A.P.D.; Momo, F.D.S.; Kim, H. A Case Study for Grain Quality Assurance Tracking based on a Blockchain Business Network 2018. In Proceedings of the Symposium on Foundations and Applications of Blockchain (FAB 18), Los Angeles, CA, USA, 9 March 2018.

35. Lei, H.; Israr, U.; Do-Hyeun, K. A Secure Fish Farm Platform Based on Blockchain for Agriculture Data Integrity, Computers and Electronics in Agriculture; arXiv: Ithaca, NY, USA, 2020; p. 170. ISSN 0168-1699.

36. Tian, F. A supply chain traceability system for food safety based on HACCP, blockchain \& Internet of things. In Proceedings of the 2017 International Conference on Service Systems and Service Management, Dalian, China, 16-18 June 2017; pp. 1-6.

37. Bumblauskas, D.; Mann, A.; Dugan, B.; Rittmer, J. A blockchain use case in food distribution: Do you know where your food has been? Int. J. Inf. Manag. 2020, 52, 102008. [CrossRef]

38. Fishcoin a Blockchain Based Data Ecosystem for the Global Seafood Industry, Fishcoin, White Paper. 2018. Available online: https://fishcoin.co/files/fishcoin.pdf (accessed on 24 May 2020).

39. Rejeb, A. Halal meat supply chain traceability based on HACCP, blockchain and internet of things. Acta Tech. Jaurinensis 2018, 11, 218-247. [CrossRef]

40. Kim, H.; Laskowski, M. Agriculture on the blockchain: Sustainable solutions for food, farmers, and financing. SSRN Electron. J. 2017, 10, 2139. [CrossRef]

41. Kumar, M.V.; Iyengar, N.C.S.N. A framework for blockchain technology in rice supply chain management plantation. Future Gener. Commun. Netw. 2017, 125-130. [CrossRef]

42. Zhang, Q.; Han, Y.-Y.; Su, Z.-B.; Fang, J.-L.; Liu, Z.-Q.; Wang, K.-Y. A storage architecture for high-throughput crop breeding data based on improved blockchain technology. Comput. Electron. Agric. 2020, 173, 105395. [CrossRef]

43. Umamaheswari, S.; Sreeram, S.; Kritika, N.; Prasanth, D.R.J. BIoT: Blockchain based IoT for Agriculture. In Proceedings of the 2019 11th International Conference on Advanced Computing (ICoAC), Chennai, India, 18-20 December 2019; pp. 324-327.

44. Awan, S.H.; Ahmed, S.; Nawaz, A.; Sulaiman, S.; Zaman, K.; Ali, M.; Najam, Z.; Imran, S. BlockChain with IoT, an emergent routing scheme for smart agriculture. Int. J. Adv. Comput. Sci. Appl. 2020, 11. [CrossRef]

45. Baralla, G.; Pinna, A.; Corrias, G. Ensure traceability in european food supply chain by using a blockchain system. In Proceedings of the 2019 IEEE/ACM 2nd International Workshop on Emerging Trends in Software Engineering for Blockchain (WETSEB), Montreal, QC, Canada; 2019; pp. 40-47.

46. Dobbins, A.; Sprinkle, A.; Hadley, B.; Cazier, J.; Wilkes, J. Blocks for bees: Solving bee business problems with blockchain technology. In Proceedings of the ARBS 2018 5th Annual Conference, Johnson City, TN, USA, 22-23 March 2018; pp. 11-16.

47. Casino, F.; Kanakaris, V.; Dasaklis, T.K.; Moschuris, S.; Rachaniotis, N.P. Modeling Food Supply Chain Traceability Based on Blockchain Technology. IFAC-PapersOnLine 2019, 52, 2728-2733. [CrossRef]

48. Shahid, A.; Almogren, A.; Javaid, N.; Al-Zahrani, F.A.; Zuair, M.; Alam, M. Blockchain-based agri-food supply chain: A complete solution. IEEE Access 2020, 8, 69230-69243. [CrossRef]

49. Wang, Z.; Liu, P. Application of blockchain technology in agricultural product traceability system. In Intelligent Tutoring Systems; Springer Science and Business Media LLC: Cham, Switzerland, 2019; pp. 81-90. 
50. Orsato, R.J.; Cohen, A. The Impact of Blockchain Technology on Eco-labelling Schemes: A Study in the Fishing Industry, Sao Paulo. 2020. Available online: http://bibliotecadigital.fgv.br/dspace/handle/10438/29033 (accessed on 28 May 2020).

51. Unurjargal, E.; Comuzzi, M. Blockchain-Supported Food Supply Chain Reference Architecture. Graduate School of UNIST, 2019. Available online: https://scholarworks.unist.ac.kr/handle/201301/25889 (accessed on 28 May 2020).

52. Mao, D.; Hao, Z.; Wang, F.; Li, H. Innovative blockchain-based approach for sustainable and credible environment in food trade: A case study in Shandong province, China. Sustainability 2018, 10, 3149. [CrossRef]

53. Ge, L.; Brewster, C.; Spek, J.; Smeenk, A.; Top, J.; Van Diepen, F.; Klaase, B.; Graumans, C.; Wildt, M.D.R.D. Blockchain for Agriculture and Food: Findings from the Pilot Study; Report 2017-112; Wageningen University and Research: Wageningen, The Netherlands, 2017.

54. Hayati, H.; Nugraha, I.G.B.B. Blockchain based traceability system in food supply chain. In Proceedings of the 2018 International Seminar on Research of Information Technology and Intelligent Systems (ISRITI), Yogyakarta, Indonesia, 21 November 2018; pp. 120-125.

55. Gunasekera, D.; Valenzuela, E. Adoption of blockchain technology in the australian grains trade: An assessment of potential economic effects. Econ. Pap. A J. Appl. Econ. Policy 2020, 39, 152-161. [CrossRef]

56. de Ruyter de Wildt, M.; van Ginkel, M.; Coppoolse, K.; van Maarseveen, B.; Walton, J.; Kruseman, G. Blockchain for Food: Making Sense of Technology and the Impact on Biofortified Seeds. Community of Practice on Socio-economic Data Report 2019. CGIAR Platform for Big Data in Agriculture. 2019. Available online: https://hdl.handle.net/10568/106615 (accessed on 29 May 2020).

57. Chan, K.Y.; Abdullah, J.; Shahid, A. A framework for traceable and transparent supply chain management for agri-food sector in malaysia using blockchain technology. Int. J. Adv. Comput. Sci. Appl. 2019, 10. [CrossRef]

58. Mohan, T. Improve Food Supply Chain Traceability using Blockchain, 2018, The Pennsylvania State University, The Graduate School College of Engineering. Available online: https://etda.libraries.psu.edu/ catalog/14913txm91 (accessed on 29 May 2020).

59. Marinello, F.; Atzori, M.; Lisi, L.; Boscaro, D.; Pezzuolo, A. Development of a traceability system for the animal product supply chain based on blockchain technology. Nantes 2017, 1, 258-268.

60. Lin, J.; Shen, Z.; Zhang, A.; Chai, Y. Blockchain and IoT based food traceability for smart agriculture. In Proceedings of the 3rd International Conference on Crowd Science and Engineering-ICCSE'18, Singapore, 28-31 July 2018; Volume 3, p. 3.

61. Mondal, S.; Wijewardena, K.; Karuppuswami, S.; Kriti, F.N.; Kumar, D.; Chahal, P. Blockchain inspired RFID-based information architecture for food supply chain. IEEE Internet Things J. 2019, 6, 5803-5813. [CrossRef]

62. Liao, Y.; Xu, K. Traceability system of agricultural product based on block-chain and application in tea quality safety management. J. Physics Conf. Ser. 2019, 1288. [CrossRef]

63. Cook, Blockchain: Transforming the Seafood Supply Chain, WWF. 2018. Available online: http://awsassets. wwfnz.panda.org/downloads/draft_blockchain_report_1_4_1.pdf (accessed on 27 May 2020).

64. Honeysuckle White expands Thanksgiving Traceable Turkey Program, Continuing its Commitment to Food Transparency, Honeysuckle White. 2018. Available online: https://www.cargill.com/2018/honeysuckle-whiteexpands-thanksgiving-traceable-turkey-program (accessed on 23 May 2020).

65. Hua, J.; Wang, X.; Kang, M.; Wang, H.; Wang, F.-Y. Blockchain based provenance for agricultural products: A distributed platform with duplicated and shared bookkeeping. 2018 IEEE Intell. Veh. Symp. (IV) 2018, 97-101. [CrossRef]

66. Biswas, K.; Muthukkumarasamy, V.; Lum, W. Blockchain Based Wine Supply Chain Traceability System. In Proceedings of the Future Technologies Conference (FTC), Vancouver, BC, Canada, 29-30 November 2017; pp. 56-62.

67. Visser, C.; Hanich, Q. How Blockchain is Strengthening Tuna Traceability to Combat Illegal Fishing. 2017. Available online: https://theconversation.com/how-blockchain-is-strengthening-tuna-traceability-to-combatillegal-fishing-89965 (accessed on 14 May 2020). 
68. Saji, A.C.; Vijayan, A.; Sundar, A.J.; Baby Syla, L. Permissioned blockchain-based agriculture network in rootnet protocol. In Proceedings of the International Conference on Innovative Computing and Communications. Advances in Intelligent Systems and Computing, Ostrava, Czech Republic, 21-22 March 2019; Khanna, A., Gupta, D., Bhattacharyya, S., Snasel, V., Platos, J., Hassanien, A., Eds.; Springer: Singapore, 2020; p. 1059.

69. Huynh, T.S.; Nguyen, L.A.T. Developing blockchain-based system for tracking the origin of chicken products. Int. J. Innov. Technol. Explor. Eng. 2019, 8, 10.

70. Scuderi, A.; Foti, V.T.; Timpanaro, G. The Supply Chain Value of POD and PGI Food Products through the Application of Blockchain. Calit. Acces Success 2019, 20, 580-587. Available online: http://hdl.handle.net/20. $500.11769 / 363300$ (accessed on 28 May 2020).

71. Malik, S.; Kanhere, S.S.; Jurdak, R. ProductChain: Scalable Blockchain Framework to Support Provenance in Supply Chains. In Proceedings of the 2018 IEEE 17th International Symposium on Network Computing and Applications (NCA), Cambridge, MA, USA, 26-28 November 2018; pp. 1-10.

72. Arsyad, A.A.; Dadkhah, S.; Köppen, M.; Dhadkah, S. Two-factor blockchain. In Lecture Notes on Data Engineering and Communications Technologies; Springer Science and Business Media LLC: Cham, Switzerland, 2018; pp. 332-339.

73. Kakkar, A.; Ruchi., A. Blockchain technology solution to enhance operational efficiency of rice supply chain for food corporation of India. In Sustainable Communication Networks and Application Lecture Notes on Data Engineering and Communications Technologies; Karrupusamy, P., Chen, J., Shi, Y., Eds.; Springer: Cham, Switzerland, 2020; p. 39.

74. Lamport, L.; Shostak, R.; Pease, M. The byzantine generals problem. ACM Trans. Program. Lang. Syst. 1982, 4, 382-401. [CrossRef]

75. Konstantopoulos, G. Understanding Blockchain Fundamentals, Part 1: Byzantine Fault Tolerance. Available online: https://medium.com/loom-network/understanding-blockchain-fundamentals-part-1-byzantinefault-tolerance-245f46fe8419 (accessed on 22 October 2019).

76. Castor, A. A (Short) Guide to Blockchain Consensus Protocols. Available online: https://www.coindesk.com/ short-guide-blockchain-consensus-protocols (accessed on 22 October 2019).

77. Massessi, D. Public vs Private Blockchain in a Nutshell. Available online: https://medium.com/coinmonks/ public-vs-private-blockchain-in-a-nutshell-c9fe284fa39f (accessed on 23 October 2019).

78. Smart Supply Chain: Farm-to-Fork Traceability for Large Scale Farming, Bühler. Available online: https://digital.buhlergroup.com/fileadmin/uploads/buhler/Digital/Images/Smartsupply/Brochure_Final_ Light.pdf (accessed on 26 May 2020).

79. George, R.V.; Harsh, H.O.; Ray, P.; Babu, A.K. Food quality traceability prototype for restaurants using blockchain and food quality data index. J. Clean. Prod. 2019, 240, 118021. [CrossRef]

80. Wattanajantra, A. How Blockchain Traceability Can Improve Supply Chain Management. Available online: https://www.sage.com/en-gb/blog/blockchain-traceability-supply-chain/\#\%20gate-2bbb8114-16b1-45189f6e-3a7dfb785d23 (accessed on 17 September 2019).

81. Gandino, F.; Montrucchio, B.; Rebaudengo, M.; Sanchez, E. On improving automation by integrating RFID in the traceability management of the agri-food sector. IEEE Trans. Ind. Electron. 2009, 56, 2357-2365. [CrossRef]

82. Cheng, C.; Jiang, P.; Liu, J. A common traceability method for agricultural products based on data center. Sens. Lett. 2013, 11, 1269-1273. [CrossRef]

83. Kim, H.M.; Laskowski, M. Towards an ontology-driven blockchain design for supply chain provenance. SSRN Electron. J. 2016. [CrossRef]

84. Tse, D.; Zhang, B.; Yang, Y.; Cheng, C.; Mu, H. Blockchain application in food supply information security. In Proceedings of the 2017 IEEE International Conference on Industrial Engineering and Engineering Management (IEEM), Singapore, 10-13 December 2017; pp. 1357-1361.

85. Latino, M.; Corallo, A.; Menegoli, M. From industry 4.0 to agriculture 4.0: How manage product data in agri-food supply chain for voluntary traceability, a framework proposed. World Acad. Sci. Eng. Technol. Int. J. Nutr. Food Eng. 2018, 12, 126-130.

86. Gálvez, J.F.; Mejuto, J.; Simal-Gandara, J. Future challenges on the use of blockchain for food traceability analysis. Trends Anal. Chem. 2018, 107, 222-232. [CrossRef] 
87. Koirala, R.C.; Dahal, K.; Matalonga, S. Supply chain using smart contract: A blockchain enabled model with traceability and ownership management. In Proceedings of the 2019 9th International Conference on Cloud Computing, Data Science \& Engineering (Confluence), Noida, India, 10-11 January 2019; pp. 538-544.

88. Creydt, M.; Fischer, M. Blockchain and more-Algorithm driven food traceability. Food Control 2019, 105, 45-51. [CrossRef]

89. IBM. About IBM Food Trust. Available online: https://www.ibm.com/downloads/cas/8QABQBDR (accessed on 24 October 2019).

90. Hyperledger: How Walmart brought unprecedented transparency to the food supply chain with Hyperledger Fabric. Available online: https://spectrum.chat/blockchain-apps/supply-chain/how-walmart-broughtunprecedented-transparency-to-the-food-supply-chain-with-hyperledger-fabric \{\}2e29b977-651d-4a04a513-09bbb8af50b7 (accessed on 15 May 2020).

91. Provenance from Shore to Plate: Tracking Tuna on the Blockchain. Available online: https://www.provenance. org/tracking-tuna-on-the-blockchain (accessed on 24 October 2019).

92. Blockchain Traceability, Transparency and Certification. Available online: http://www.smartagrifood.it/ index-en.html\# (accessed on 24 October 2019).

93. Pearson, S.; May, D.; Leontidis, G.; Swainson, M.; Brewer, S.; Bidaut, L.; Frey, J.G.; Parr, G.; Maull, R.; Zisman, A. Are distributed ledger technologies the panacea for food traceability? Glob. Food Secur. 2019, 20, 145-149. [CrossRef]

94. Behnke, K.; Janssen, M.F.W.H.A. Boundary conditions for traceability in food supply chains using blockchain tchnology. Int. J. Inf. Manag. 2019, 52, 101969. [CrossRef]

95. Kamilaris, A.; Fonts, A.; Prenafeta, F.; Prenafeta, F. The rise of blockchain technology in agriculture and food supply chains. Trends Food Sci. Technol. 2019, 91, 640-652. [CrossRef]

(C) 2020 by the authors. Licensee MDPI, Basel, Switzerland. This article is an open access article distributed under the terms and conditions of the Creative Commons Attribution (CC BY) license (http://creativecommons.org/licenses/by/4.0/). 